\title{
Microorganismos y hábitos de higiene. ¿Se aprende más en la Educación Infantil mediante fichas?
}

\author{
María Antonia López-Luengo \\ Área de Didáctica de las Ciencias Experimentales, Facultad de Educación de Segovia, Universidad de \\ Valladolid.Segovia.España.mariaantonia.lopez@uva.es \\ Esther González Díaz (D), Esther Paños (D) \\ Area de Didáctica de las Ciencias Experimentales, Facultad de Educación, Universidad de Castilla-La \\ Mancha.Albacete.España.esther.gonzalez@alu.uclm.es,esther.panos@uclm.es \\ José-Reyes Ruiz-Gallardo (i) \\ Área de Didáctica de las Ciencias Experimentales, Facultad de Educación, Grupo de investigación en \\ Etnobiología, Botánica y Educación, Instituto Botánico, Universidad de Castilla-La Mancha. Albacete. \\ España.josereyes.ruiz@uclm.es
}

[Recibido: 11 abril 2020. Revisado: 28 julio 2020. Aceptado: 30 septiembre 2020]

Resumen: En este trabajo se presentan los hábitos de higiene y el conocimiento que tiene un conjunto de 69 alumnos de Educación Infantil (4-6 años) sobre microorganismos y se analiza la posible influencia sobre ellos de dos propuestas didácticas diferentes (tradicional -grupo control- y con indagación -grupo experimental-). La información se obtuvo en tres momentos diferentes -pre-intervención didáctica, post intervención y dos meses después-, mediante dibujos de los niños, un cuestionario aplicado oral e individualmente con preguntas cerradas y abiertas y un cuestionario dirigido a las familias. Se detectan indicios de que el alumnado reconoce a los microorganismos como seres vivos. Tras la intervención no se encuentran diferencias estadísticamente significativas entre el grupo experimental y el control. Dos meses después se observa una mejora en el grupo control respecto a algunos hábitos de higiene y en el grupo experimental se recuerda el proceso de indagación con placas de Petri. Estos resultados abren nuevos interrogantes.

Palabras clave: Educación Infantil; Educación Científica; Indagación; Microorganismo; Hábitos de higiene.

Microorganisms and hygiene habits: Is there more to be learned in Infant Education by means of worksheets?

Abstract: The hygiene habits and the knowledge that a group of 69 students of Early Childhood Education (4-6
years old) have about microorganisms is presented in this work. The possible influence on them of two different
didactic proposals (traditional -control group- and inquiry-based learning -experimental group-) is analysed. The
information was obtained at three different moments -pre didactic intervention, post intervention and two
months later-. The used tolls were children's drawings, an oral and individually applied questionnaire with closed
and open questions and a questionnaire addressed to the families. Indications are detected that the students
recognize the microorganisms as living beings. After the intervention, no statistically significant differences were
found between the experimental and control groups. Two months later an improvement was observed in the
control group with respect to some hygiene habits and in the experimental group the process of investigation
with petri dishes was recalled. These results open new research questions. Keywords: Early Childhood Education; Science Education; IBSE; Micro-organism; Hygiene

Para citar este artículo: López Luengo M. A., González Díaz E., Paños E. y Ruiz Gallardo J. R. (2021) Microorganismos y hábitos de higiene. ¿Se aprende más en la Educación Infantil mediante fichas?. Revista Eureka sobre Enseñanza y Divulgación de las Ciencias 18(2), 2302.doi: 10.25267/Rev_Eureka_ensen_divulg_cienc.2021.v18.i2.2302 


\section{Introducción}

En los últimos quince años ha aumentado mucho el interés hacia la educación científica durante la etapa infantil. Simultáneamente, la indagación se ha introducido como una de las estrategias más exitosas para la educación científica y, desde EE. UU., se ha extendido el concepto de educación STEAM. Todo ello está enfocado hacia los grandes retos que enfrenta la sociedad del siglo XXI y demanda una reflexión seria, fundamentada en el análisis científico de las prácticas educativas escolares.

El trabajo que aquí se presenta pretende contribuir a dicha reflexión generadora de conocimiento sobre la enseñanza-aprendizaje científico en Educación Infantil (EI). Su objetivo fundamental es averiguar si, tanto los hábitos de higiene que tiene el alumnado de EI como su percepción inicial sobre los microorganismos, evolucionan de modo diferente cuando se emplea en el aula una metodología tradicional transmisiva con la realización de fichas u otra con indagación en la que estas no se realizan. Se busca también conocer la permanencia en el tiempo de los aprendizajes mediante un estudio longitudinal a 2 meses.

\section{Los microorganismos}

Más allá de su enorme interés científico-tecnológico y económico (biorremediación, producción de alimentos y fármacos, etc.), el estudio de los microorganismos tiene un gran interés didáctico: ilustra muy bien las relaciones Ciencia-Tecnología-Sociedad-Ambiente a lo largo de la historia y es fundamental para el entendimiento y la aplicación de conceptos científicos clave como la diferenciación entre ser vivo y materia inerte. Por ello, es importante la promoción escolar, desde las primeras etapas, de una visión real y equilibrada de los microorganismos, facilitadora de reflexión y generadora de aprendizajes que se reflejen en actuaciones consecuentes.

Sin embargo, a pesar de que su conocimiento puede ser una herramienta imprescindible para los niños en la comprensión e interpretación de hechos cotidianos (producción y conservación de alimentos, higiene, vacunación, etc.), es un contenido que apenas está presente en el currículo español de Educación Primaria (Real Decreto 126/2014) y cuyo tratamiento en los libros de texto es muy limitado (Ballesteros, Paños y Ruiz-Gallardo 2018). Este problema ha sido detectado y analizado también en otros países (Bandiera 2007, Byrne 2011, Vijapurkar y Konde 2014, Mafra, Lima y Carvalho 2015).

Como puede esperarse, carece de referencias directas en el currículo de EI (Real Decreto 114/2004), pero puede relacionarse fácilmente con contenidos que sí están presentes tanto en el Área del Conocimiento del entorno -la diferenciación entre los seres vivos y la materia inerte o la importancia de otros seres vivos en nuestra vida-, claramente concerniente a la ciencia, como en el Área de Conocimiento de sí mismo y autonomía personal -la práctica de hábitos saludables o de higiene-. La adquisición de hábitos de higiene durante los primeros años tiene consecuencias en la salud individual y social; requiere de un trabajo coordinado entre la escuela y la familia. Para un mayor éxito en el proceso, se recomienda insistir en el porqué de la higiene, lo cual conecta directamente con el conocimiento científico.

Diversos autores han estudiado la comprensión del alumnado sobre la relación de los microorganismos con el ser humano y el papel que desempeñan en la naturaleza, pero son muy pocos los trabajos que incluyen alumnado menor de diez años (Kalish 1996, Byrne 2011, Prokop, Fančovičová y Krajčovičová 2016, Dominguez et al. 2018, Ruiz-Gallardo y Paños 2018). Según los estudios realizados, el alumnado de 4-7 años mayoritariamente no considera a los microorganismos como seres vivos (Garrido 2017) y desconoce el papel que realizan en los ecosistemas o sus aplicaciones tecnológicas (Byrne y Sharp 2006, Trivelato 1995 referido por Dominguez et al. 2018), aunque su experiencia con la enfermedad podría generar una idea más 
adecuada del vínculo entre los microorganismos y la salud (Prokop, Fančovičová y Krajčovičová 2016).

\section{Enseñanza de las ciencias en Educación Infantil}

Consideramos que la educación científica debe iniciarse en la etapa de EI, dado que los contenidos científicos -como pueden ser los relativos a la microbiología señalados más arribano solo favorecen el desarrollo cognitivo (Carver y Shrager 2012, COSCE 2011, Eshach 2011, Eshach y Fried 2005, French 2004, Watters, Diezmann, Grieshaber y Davis 2000), sino que son centros de interés alrededor de los cuales se construyen más fácilmente aprendizajes de varios tipos con repercusión en el futuro (Saçkes, Trundle, Bell y O'Connell 2011).

De modo generalizado, en EI se defiende el enfoque globalizado, así como el empleo de actividades manipulativas, experiencias directas y pruebas de ensayo error, por considerarse altamente motivadoras y facilitadoras de la adquisición de conocimientos y destrezas. Sin embargo, las ciencias están poco presentes en las aulas de EI y a menudo están sujetas a fichas y a proyectos editoriales (Saçkes, et al., 2011).

Cuando el foco se encuentra en la enseñanza-aprendizaje de las ciencias, se insta al profesorado a guiar paso a paso al alumnado en la realización de sus investigaciones (Yoon y Onchwari 2006). Mediante preguntas, sugerencias y ayudas en la realización de las experiencias, los docentes deben facilitar el desarrollo de las competencias y conocimientos del alumnado (Cruz-Guzmán, García-Carmona y Criado 2017). Si las actividades de aula propician el razonamiento y la transferencia, será más factible que el alumnado desarrolle habilidades científicas tales como: observar, formular hipótesis, extraer conclusiones, reflexionar y una necesaria actitud crítica (Gerde, Schachter y Wasik 2013, Gómez-Motilla y Ruiz-Gallardo 2016); en definitiva, que adquiera competencia científica ahora y en el futuro (Eshach y Fried 2005, Froschauer 2016).

Dado el aún incipiente conocimiento sobre el efecto de la indagación en el aprendizaje de la ciencia en la etapa de EI, así como sobre la permanencia de los aprendizajes, se diseñó la experimentación que se presenta; centrada en un tema científico relevante pero poco frecuente en las primeras etapas educativas.

\section{Metodología}

\section{Diseño}

En este trabajo se concibe a los niños como poseedores de conocimiento teórico, conceptual y procedimental en el que la acción educativa influye. No se trata de averiguar la comprensión infantil de las causas de la enfermedad como ejemplo potencial de razonamiento biológico teórico (Kalish 1996, Sigelman y Glaser 2019), ni de obtener un solo modelo coherente y global sobre el significado de ser vivo, sino que se atiende a un fragmento concreto (término microorganismo y su campo semántico) y se busca coherencia parcial (aplicaciones tecnológicas e higiene), al tiempo que se trata de inferir el efecto del trabajo de aula sobre el conocimiento infantil.

Para poder comprobar si la metodología empleada en el aula de EI influye en el conocimiento sobre los microorganismos y en la evolución de los hábitos de higiene, se realizó una investigación cuasi-experimental y longitudinal, con diseño pre-post. En ella se analiza el conocimiento infantil previo a la unidad didáctica, al término de esta y dos meses después. La intervención didáctica sigue las susodichas recomendaciones metodológicas para EI; la diferencia entre la secuencia de actividades del grupo experimental y del grupo control se encuentra en que en la primera se incluye la indagación (Anexo 1). 


\section{Muestra}

Los participantes en este estudio son 69 alumnos, 34 de 4 años y 35 de 5 años (Tabla 1) de una población rural (Chinchilla de Monte Aragón -Albacete-). La muestra tiene un carácter no probabilístico y de conveniencia. Se estableció aleatoriamente una de las dos aulas de cada edad como grupo control y otra como grupo experimental. En el estudio participaron aquellos niños con necesidades educativas especiales pertenecientes a los distintos grupos.

Tabla 1. Distribución general de los participantes, por grupo (control y experimental), sexo y curso.

\begin{tabular}{|c|c|c|c|c|}
\hline \multirow[t]{2}{*}{ Grupo } & \multicolumn{2}{|c|}{4 años } & \multicolumn{2}{|c|}{5 años } \\
\hline & Masc. & Fem. & Masc. & Fem. \\
\hline Experimental & 9 & 8 & 7 & 11 \\
\hline Control & 9 & 8 & 6 & 11 \\
\hline Subotal & 18 & 16 & 13 & 22 \\
\hline Total & \multicolumn{2}{|c|}{34} & \multicolumn{2}{|c|}{35} \\
\hline
\end{tabular}

\section{Proceso de recogida de datos e instrumentos empleados}

El tipo de preguntas y la forma de intervención en el proceso de obtención de datos es clave en la investigación con niños. La entrevista es un gran método de obtención de datos reales y fiables (Kvale 2011), sin embargo, no es sencillo, especialmente con niños, dado que existen problemas de tipo ético y metodológico en su realización (Westcott y Littleton, 2005; Gallegos-Cázares et al. 2017). Por todo ello, en este trabajo se utilizaron tres instrumentos.

En primer lugar, en busca de los referentes de los niños (Vivaldi y Salsa 2017), se les facilitó una hoja en blanco y se les pidió que dibujasen microorganismos donde se encuentran normalmente. Así, la toma de datos queda incluida en una unidad didáctica globalizada que se aproxima a la educación STEAM puesto que trabaja de modo integrado aspectos de educación en la expresión artística (dibujo, volumen, música y ritmo) al tiempo que aborda contenidos científicos (microorganismos, su estudio y su relación con los seres humanos), de ingeniería (planificación y estudio de procesos -secuencia de pasos para una correcta higiene de manos y dientes, elaboración de pan-) y tecnológicos (fabricación de pan) (Anexo 1). Al quedar incluido este instrumento en la dinámica de aula el alumnado lo realizó en las mesas de trabajo junto a sus compañeros habituales.

En segundo lugar, se aplicó un cuestionario oral e individualmente. Este instrumento estaba constituido por siete preguntas cerradas relacionadas con los hábitos de higiene bucal y de manos, con tres posibles respuestas cada una (siempre, a veces, nunca), basado en Moreno (2015). Además, se preguntaba al alumnado si conocía los términos microorganismo, microbio, virus, hongo y bacteria y, en caso de conocer alguno de ellos, qué es lo que sabía. Todo el proceso fue realizado por la misma persona, quien, en un tiempo razonable -tanto para los niños como para la dinámica de aula- y sin necesidad de grabación, tomó nota textual de lo expresado por cada individuo en una ficha independiente (Anexo 2).

Ambos instrumentos -dibujos y cuestionario- se emplearon 3 veces: antes de la intervención didáctica, al término de esta y trascurridos dos meses desde su finalización.

El tercer instrumento consistió en un cuestionario dirigido a las familias, formado por preguntas cerradas sobre los hábitos higiénicos y un campo abierto para observaciones. Su objeto era evaluar la transferencia del conocimiento escolar a la vida cotidiana y la permanencia de los aprendizajes, por ello, se facilitó al alumnado en el momento de la tercera aplicación del cuestionario para que lo llevasen a casa y lo trajeran cumplimentado (Anexo 3). 


\section{Análisis de datos}

Los datos proporcionados por todos los instrumentos se volcaron en una matriz. Las respuestas sobre hábitos higiénicos: Siempre, A veces y Nunca se codificaron respectivamente con los valores 1,2 y 3 . Las respuestas afirmativas correspondientes al conocimiento de la terminología microbiológica se codificaron con el valor 1 , las negativas con el valor 0 . Igual procedimiento se siguió con el cuestionario dirigido a las familias.

Los datos pre-intervención didáctica se analizaron mediante estadística descriptiva. Para detectar cambios tras la intervención en ambos grupos se aplicaron diferentes estadísticos de contraste. Debido al carácter ordinal de los datos se emplearon índices estadísticos de tipo no paramétrico, no obstante, se calculó la media estadística para facilitar la interpretación. Así, para el contraste intragrupo (muestras relacionadas), y para datos politómicos, con medidas repetidas, se utilizó el estadístico de Friedman (representado por $\chi^{2}$ (ji cuadrado) con post-hoc mediante el estadístico de Wilcoxon. Para los datos dicotómicos, se aplicó la $Q$ de Cochran con post-hoc mediante la prueba de McNemar.

El contraste entre el grupo control y el experimental se realizó mediante el estadístico de Mann-Withney $(U)$ en datos politómicos y para los dicotómicos se emplearon tablas de cruce con la prueba $\chi^{2}$.

Lo que decían saber de los microorganismos se recogió en una columna de datos; mientras que los dibujos generaron cuatro columnas: descripción del dibujo, ubicación de los microorganismos, color utilizado y forma. El análisis detallado de estos datos supera la extensión de este artículo, no obstante, se presenta una aproximación a la triangulación del análisis estadístico del cuestionario. Se omite el análisis estadístico de las distintas dimensiones de los dibujos porque, al no haberse realizado de modo aislado, el dibujo de unos niños pudo haber influido sobre el trabajo de otros.

\section{Resultados y discusión}

No todos los niños realizaron los dibujos en los tres momentos, por lo que se obtuvieron datos completos de 57 escolares, lo que supone un total de 171 dibujos y 207 registros de cuestionarios.

\section{Análisis exploratorio (Pre-intervención)}

Los hábitos de higiene más frecuentes entre el alumnado aparecen en la Tabla 2. Se señala, además, que casi la mitad $(47,83 \%)$ reconoce lavarse siempre los dientes por la mañana y después de cada comida. La mayoría $(84,06 \%)$ declara usar jabón cuando se lava las manos.

Tabla 2. Frecuencia de lavado de manos y dientes en los participantes antes de la intervención (preguntas 3 y 7)

\begin{tabular}{llll}
\hline & Siempre & A veces & Nunca \\
\hline $\begin{array}{c}\text { Frecuencia lavado manos } \\
\text { después de hacer pis }\end{array}$ & $(57,97 \%)$ & $(21,74 \%)$ & $(20,29 \%)$ \\
$\begin{array}{c}\text { Frecuencia lavado dientes } \\
\text { antes de dormir }\end{array}$ & $(65,22 \%)$ & $(23,19 \%)$ & $(11,59 \%)$ \\
\hline
\end{tabular}

Al igual que lo hallado en trabajos previos realizados en edades superiores, los términos microbio y microorganismo son desconocidos para la mayor parte de los escolares $(66,66 \%)$. Un $34,78 \%$ afirma haber escuchado antes la palabra virus y un $44,93 \%$ la palabra bacterias. Tan solo un $17,39 \%$ ha oído hablar antes de hongos y solo uno lo relaciona con su experiencia: «Que te pica». 
Entre quienes conocen los términos microbio o microorganismo, un 55,07\% es capaz de dar una descripción (Tabla 3). La mayoría dice que son «bichos» y algunos concretan que hacen daño: «Un microbio es un bicho que se te mete en nuestro cuerpo y nos sienta mal»; lo que confirma el carácter negativo que se otorga a los términos bicho (Portela 2013) y microbio (Mayerhofer 2009). Un par de niños identifican los microbios con artrópodos: «Microbios son las arañas y los piojos». En la categoría Otros se incluyen las palabras «cosa» y «micrófono», empleada esta última por dos niños, posiblemente porque les suenan parecido. Un 8,7\% de los niños equiparan estos términos con "guisantes», algo esférico, lo que coincidiría con lo encontrado por Prokop, Fančovičová y Krajčovičová (2016). Para unos pocos es repulsivo $(1,45 \%):$ «Un microbio da asco».

Tabla 3. Palabras que los participantes otorgan a los microbios.

\begin{tabular}{lcc}
\hline Categoría & $\mathbf{n}$ & $\mathbf{\%}$ \\
\hline Bichos-insectos & 30 & 43,48 \\
Otros & 8 & 11,59 \\
Sin contestar & 31 & 44,93 \\
Total & 69 & 100 \\
\hline
\end{tabular}

Los niños que reconocen la palabra virus la identifican o asocian con enfermedad: «Virus son bichos que dan fiebre y dolores de cabeza»; «Virus es el de la tos»; «Virus es que estás malito»; algo que se ajusta a los resultados de Mafra et al. (2015), Jones y Rua. (2006), Dominguez et al. (2018) y Ballesteros, Paños y Ruiz-Gallardo (2018) y reflejaría la información recibida en casa.

Los alumnos que declaran conocer la palabra bacteria hablan de algo que está en los dientes $(7,24 \%)$, en las manos (4,35\%) y en la barriga (2,90\%). El 32,26\% de los alumnos relaciona estos seres con causa de enfermedades: «Bacterias son bichos que hacen lo mismo, hacen daño en la barriga o donde se metan». Ninguna explicación les atribuye utilidades o beneficios.

De modo coherente, los dibujos pre-intervención muestran la casi carencia de referentes, no hay formas o patrones característicos y definidos entre los niños de 4 años salvo el de bicho (artrópodo). Aparecen de manera mayoritaria y general formas multicolores, pero en algunos casos son de tipo artrópodo o monstruo de cara amenazante, también aparece una niña enferma. Según ha sido descrito (Armitage y Allen 2015) el dibujo es realista mientras haya señales de semejanza, si estas fallan, por desconocimiento, el dibujo remite a la intención. De igual modo los niños de 5 años representan mayoritariamente esferas con o sin cilios a menudo con cara sonriente, resultado concordante con lo obtenido por Prokop, Fančovičová y Krajčovičová (2016). En contra de los hallazgos de estos autores, en este grupo de edad los microorganismos se relacionan con el cuerpo humano: aparecen bastantes manos, algunas de ellas muestran manchas negras y marrones y en algún caso esferas sonrientes. Son también abundantes los seres tipo artrópodo que responderían al referente bicho, en algún caso incluso se sitúa los microbios en el pelo (¿piojos?). Según los dibujos, los microorganismos para los más pequeños no tienen una ubicación fija y definida. Estos resultados concuerdan parcialmente con los hallados en estudios previos y edades superiores (6-16 años), según los cuales estos seres vivos se relacionan exclusivamente con suciedad, perjuicios para los humanos y causa de enfermedades (Luna y Solís 1997, Jones y Rua 2006, Bandiera 2007, Byrne, Grace y Hanley 2009, Ballesteros, Paños y Ruiz-Gallardo 2018).

A pesar de que se ha señalado que entre 4 y 7 años no se reconoce a los microbios como seres vivos (Garrido 2017), la representación de los microorganismos como artrópodos y esferas 
peludas con caras sonrientes o amenazadoras podría significar que se les considera seres vivos. Sin olvidar el animismo infantil (Piaget 2008), hay que tener en cuenta que la intención con la que se crea el dibujo puede variar su significado, en este caso su intención es comunicativa (Vivaldi y Salsa 2017). La diferenciación temprana de los seres vivos de los objetos inertes podría estar basada en la asignación a los primeros de intencionalidad (Carey 1985). Expresiones como la siguiente: «Microbio es una cosa que se te mete en el cuerpo, un bicho», indicarían que quien la emite reconoce a los microbios en la categoría ontológica de ser vivo.

\section{Efecto de la intervención del grupo experimental}

Respecto a los hábitos de higiene, apenas aparecen cambios tras la intervención y, en algunos casos, parece que empeoran (Tabla 4). No obstante, las diferencias no son estadísticamente significativas en ninguna de las preguntas analizadas, por lo que puede afirmarse que los hábitos de higiene del grupo experimental no se han modificado.

Tabla 4. Cambios sufridos en los hábitos de higiene. Grupo experimental.

\begin{tabular}{|c|c|c|c|c|c|c|c|c|c|c|c|c|}
\hline & \multicolumn{3}{|c|}{ Pregunta 1} & \multicolumn{3}{|c|}{ Pregunta 2} & \multicolumn{3}{|c|}{ Pregunta 3} & \multicolumn{3}{|c|}{ Pregunta 4} \\
\hline & $\operatorname{Pr}$ & P1 & P2 & $\operatorname{Pr}$ & P1 & P2 & $\operatorname{Pr}$ & P1 & P2 & $\operatorname{Pr}$ & P1 & P2 \\
\hline$X$ & 1,46 & 1,46 & 1,29 & 1,60 & 1,57 & 1,37 & 1,57 & 1,66 & 1,43 & 1,11 & 1,00 & 1,03 \\
\hline $\mathrm{dT}$ & 0,51 & 0,61 & 0,46 & 0,78 & 0,78 & 0,60 & 0,74 & 0,73 & 0,61 & 0,32 & 0,00 & 0,17 \\
\hline \multirow[t]{3}{*}{$\chi^{2}$} & \multicolumn{3}{|c|}{$3,10^{\mathrm{ns}}$} & \multicolumn{3}{|c|}{$2,26^{\mathrm{ns}}$} & \multicolumn{3}{|c|}{$1,51^{\mathrm{ns}}$} & \multicolumn{3}{|c|}{$5,20^{\mathrm{ns}}$} \\
\hline & \multicolumn{4}{|c|}{ Pregunta 5} & \multicolumn{4}{|c|}{ Pregunta 6} & \multicolumn{4}{|c|}{ Pregunta 7} \\
\hline & \multicolumn{2}{|l|}{$\operatorname{Pr}$} & P1 & P2 & $\operatorname{Pr}$ & \multicolumn{2}{|c|}{$\mathrm{P} 1$} & P2 & $\operatorname{Pr}$ & \multicolumn{2}{|c|}{ P1 } & P2 \\
\hline$X$ & \multicolumn{2}{|l|}{1,69} & 1,6 & 1,66 & 1,54 & \multicolumn{2}{|c|}{1,31} & 1,46 & 1,43 & \multicolumn{2}{|c|}{1,26} & 1,29 \\
\hline $\mathrm{dT}$ & \multicolumn{2}{|l|}{0,8} & 0,74 & 0,80 & 0,61 & \multicolumn{2}{|c|}{0,63} & 0,66 & 0,74 & \multicolumn{2}{|c|}{0,51} & 0,46 \\
\hline$\chi^{2}$ & \multicolumn{3}{|c|}{$0,10^{\mathrm{ns}}$} & & \multicolumn{4}{|c|}{$3,88^{\mathrm{ns}}$} & & \multicolumn{2}{|c|}{$0,91^{\mathrm{ns}}$} & \\
\hline
\end{tabular}

Pr: evaluación previa; P1: primer post-test (inmediatamente después de la experiencia); P2: segundo post-test

(2 meses después de la experiencia); X: media; dT: desviación típica; $\chi^{2}$ : ji cuadrado; ns: no significativo

En cuanto a las palabras relacionadas con los microorganismos que los niños afirman reconocer, en este grupo solo aparecen diferencias estadísticas en las palabras microorganismo y microbio; aunque, como se observa en la Tabla 5 , se trata de una aguda reducción en el concepto de microorganismo entre el primer y el segundo pos-test, incluso por debajo del valor de origen. En el caso de microbio sí hay una mejora en el concepto tras la intervención, que no parece declinar en el tiempo. Las expresiones que utilizan los niños relacionan los microbios con la enfermedad: «Los microbios están en el estómago, pues cuando estás malito o tienes tos» y, lo que resulta especialmente relevante, con la falta de higiene «Son cuando no se lavan las manos y los dientes. Hongos son cuando no se lavan las manos. Bacterias son cuando no se lavan los dientes».

De los dibujos post-intervención puede deducirse el efecto de esta: la gran mayoría de niños de ambas edades muestran dientes y manos con puntos negros o manchas de colores, en varios casos los microorganismos se sitúan en una placa de Petri. Hay un caso en el que se 
dibuja un punto negro en la tripa de un niño. Es destacable que entre los niños de 4 años siguen apareciendo muchas esferas, pero aparece un nuevo referente que sustituye a los artrópodos, el bacteriófago. En algún caso no se sitúan los microorganismos en el cuerpo sino en papeleras o contenedores de basura. Aparece un caso de un tarro con bolitas en su interior (¿levadura?). Se ha afirmado que el alumnado localiza a los microorganismos mayoritariamente en lugares sucios, como la basura (Dominguez et al. 2018, Jones y Rua 2006). Los datos aportados aquí muestran que los niños tras la intervención didáctica sitúan a los microbios en la basura y en el suelo, pero mayoritariamente en el cuerpo humano, asociados a las enfermedades y la necesidad de higiene (lavado de dientes y manos).

Tabla 5. Cambios sufridos en las respuestas de los niños ante la pregunta: ¿Has oído alguna vez la palabra microorganismo/ microbio/ virus/ hogos / bacterias?

\begin{tabular}{|c|c|c|c|c|c|c|c|c|c|c|c|c|c|c|c|}
\hline \multicolumn{16}{|c|}{ CONTROL } \\
\hline & \multicolumn{3}{|c|}{ Microorganismo } & \multicolumn{3}{|c|}{ Microbio } & \multicolumn{3}{|c|}{ Virus } & \multicolumn{3}{|c|}{ Hongos } & \multicolumn{3}{|c|}{ Bacterias } \\
\hline & $\operatorname{Pr}$ & P1 & $\mathrm{P} 2$ & $\operatorname{Pr}$ & P1 & P2 & $\operatorname{Pr}$ & P1 & P2 & $\operatorname{Pr}$ & P1 & P2 & $\operatorname{Pr}$ & P1 & $\mathrm{P} 2$ \\
\hline N1 & 6 & 23 & 8 & 12 & 32 & 29 & 14 & 27 & 22 & 2 & 16 & 5 & 3 & 22 & 16 \\
\hline$N$ & & 34 & & & 34 & & & 34 & & & 34 & & & 34 & \\
\hline$Q$ & & $20.7^{* * *}$ & & & $29.1 * *$ & & & $17.7^{* * *}$ & & & $16.3^{* * *}$ & & & $19.5^{* * *}$ & \\
\hline$M_{c \text { Pr-P1 }}$ & & $* * *$ & & & $* * *$ & & & $* *$ & & & $* * *$ & & & $* * *$ & \\
\hline$M c_{\mathrm{Pr}-\mathrm{P} 2}$ & & ns & & & $* * *$ & & & ns & & & ns & & & $* *$ & \\
\hline$M c_{\mathrm{P} 1-\mathrm{P} 2}$ & & $* *$ & & & ns & & & ns & & & ns & & & $\mathrm{ns}$ & \\
\hline$O R_{\mathrm{Pr}-\mathrm{P} 1}$ & & 0.06 & & & 0.05 & & & 0.07 & & & $\$$ & & & 0.05 & \\
\hline$O R_{\text {Pr-P2 }}$ & & & & & 0.11 & & & & & & & & & 0.13 & \\
\hline OR P1-P2 & & 8.5 & & & & & & & & & & & & & \\
\hline
\end{tabular}

EXPERIMENTAL

\begin{tabular}{|c|c|c|c|c|c|c|c|c|c|c|c|c|c|c|c|}
\hline & \multicolumn{3}{|c|}{ Microorganismo } & \multicolumn{3}{|c|}{ Microbio } & \multicolumn{3}{|c|}{ Virus } & \multicolumn{3}{|c|}{ Hongos } & \multicolumn{3}{|c|}{ Bacterias } \\
\hline & $\operatorname{Pr}$ & P1 & P2 & $\operatorname{Pr}$ & P1 & P2 & $\operatorname{Pr}$ & P1 & $\mathrm{P} 2$ & $\operatorname{Pr}$ & P1 & P2 & $\operatorname{Pr}$ & P1 & P2 \\
\hline N1 & 14 & 22 & 9 & 15 & 26 & 25 & 20 & 13 & 18 & 12 & 13 & 11 & 19 & 16 & 16 \\
\hline$N$ & & 35 & & & 35 & & & 35 & & & 35 & & & 35 & \\
\hline Q & & $9.23^{* *}$ & & & $9.6^{* *}$ & & & $2.8^{\mathrm{n}}$ & & & $0.28^{\mathrm{n}}$ & & & $0.67^{\mathrm{ns}}$ & \\
\hline$M c_{\mathrm{Pr}-\mathrm{P} 1}$ & & & ns & & $*$ & & & & & & & & & & \\
\hline$M_{c \text { Pr-P2 }}$ & & & ns & & ns & & & & & & & & & & \\
\hline$M_{c \mathrm{P} 1-\mathrm{P} 2}$ & & & $* *$ & & ns & & & & & & & & & & \\
\hline$O R_{\text {Pr-P1 }}$ & & & & & 0.21 & & & & & & & & & & \\
\hline \multicolumn{16}{|l|}{$O R_{\text {Pr-P2 }}$} \\
\hline$O R_{\text {P1-P2 }}$ & & & 14 & & & & & & & & & & & & \\
\hline
\end{tabular}

Pr: evaluación previa; P1: postest 1 (inmediato tras la experiencia); P2: postest 2 (dos meses después de la experiencia); N: número de individuos participantes; N1: número de participantes que responden "sî"; $Q:$ valor de la prueba de Cochran; Mc: Prueba de McNemar, que al no arrojar valor, se incluye solo el nivel de significatividad; OR: tamaño del efecto; ns: No significativo; ${ }^{*} \mathrm{p}<.017 ;{ }^{* *} \mathrm{p}<.01 ;{ }^{* * *} p<0.001$; $\$$ : no se puede calcular debido a que alguna casilla de la tabla de cruce es igual a 0.

En la tercera recogida de datos, los dibujos de los niños de 4 años pierden la ubicación en dientes y manos, así como en placas de Petri, en los de los niños de 5 años se mantienen. La representación del bacteriófago se mantiene en ambos grupos de edad. 


\section{Efecto de la intervención en el grupo control}

En referencia a los hábitos de higiene, en el grupo control se observan diferencias estadísticamente significativas en las preguntas 1 y 5 (Tabla 6). En la pregunta 1, correspondiente al hábito de lavarse las manos antes de comer se advierte un deterioro entre la primera y la segunda toma de datos. Sin embargo, mejora significativamente en la tercera toma de datos. En la cuestión 5, relativa al lavado de dientes al levantarse, este hábito empeora significativamente tras la intervención, aunque dos meses después, mejora de forma significativa y vuelve a una situación similar a la inicial.

El vocabulario del grupo control mejora significativamente inmediatamente después de la intervención didáctica en todos los individuos (Tabla 6), aunque en la tercera aplicación del cuestionario las palabras microorganismo, virus, hongos y bacterias tienen los mismos valores que en la primera. Solo la palabra microbio se mantiene en el tiempo y aparecen expresiones que relacionan varios términos como las siguientes: «Los microbios son bacterias»; «La levadura es un hongo». Tras la intervención aparecen dos casos que incluyen impresiones positivas, pero no aparecen dos meses después: «Son bichos que cuando se meten en tu cuerpo tienes dolor de cabeza y angustia. Los bichitos buenos están en la levadura»; «Son bichitos pequeños que cuando se meten tu cuerpo te pones enfermo. Los bichitos buenos están en el pan». El dibujo de uno de estos niños recoge precisa y exclusivamente la acción de la levadura. Hay algunos otros dibujos que representan los microorganismos como bolas dentro de botes o tarros.

Tras la intervención, la mayoría de los niños de ambas edades dibujan manos con alguna mancha encima o alrededor. Es poco frecuente la representación de los dientes sucios. Algunos los sitúan en el suelo o en el aire. Los microbios se representan generalmente mediante bolas, aunque también aparecen algunos insectoides y fagos.

Dos meses después, abundan más las representaciones de manos y dientes. Las de manos aparecen mucho junto a un grifo y una pastilla de jabón. Los microorganismos están representados como insectos, fagos, bolas, manchas o pelusas.

Tabla 6. Cambios sufridos en los hábitos de higiene. Grupo control

\begin{tabular}{|c|c|c|c|c|c|c|c|c|c|c|c|c|}
\hline & \multicolumn{3}{|c|}{ Pregunta 1} & \multicolumn{3}{|c|}{ Pregunta 2} & \multicolumn{3}{|c|}{ Pregunta 3} & \multicolumn{3}{|c|}{ Pregunta 4} \\
\hline & $\operatorname{Pr}$ & P1 & P2 & $\operatorname{Pr}$ & P1 & P2 & $\operatorname{Pr}$ & P1 & P2 & $\operatorname{Pr}$ & P1 & P2 \\
\hline$X$ & 1,53 & 1,21 & 1,71 & 1,74 & 1,59 & 1,79 & 1,68 & 1,65 & 1,82 & 1,26 & 1,09 & 1,06 \\
\hline$D T$ & ,66 & ,41 & ,63 & ,83 &, 78 &, 77 & ,88 &, 81 &, 80 &, 57 & ,29 & 0,24 \\
\hline$\chi^{2}$ & \multicolumn{3}{|c|}{$17,69^{\star *}$} & \multicolumn{3}{|c|}{$2,36^{\mathrm{ns}}$} & \multicolumn{3}{|c|}{$2,74^{\mathrm{ns}}$} & \multicolumn{3}{|c|}{$4,90^{\mathrm{ns}}$} \\
\hline$Z_{P r P P 1}(r)$ & \multicolumn{3}{|c|}{$-3,05^{*} \quad(0,52)$} & & & & & & & & & \\
\hline$Z_{P 1-P 2}(r)$ & \multicolumn{3}{|c|}{$-3,71^{* *}(-0,63)$} & & & & & & & & & \\
\hline$Z_{P r-P 2}(r)$ & \multicolumn{3}{|c|}{$-1,5^{\text {ns }}$} & & & & & & & & & \\
\hline
\end{tabular}

Pr: evaluación previa; P1: postest 1 (inmediato tras la experiencia); P2: postest 2 (dos meses después de la experiencia); $X$ : media; DT: desviación típica; $\chi^{2}$ : estadístico ji-cuadrado; $Z$ : aproximación a lo normal; $r$ : tamaño del efecto, ns: no significativo; ${ }^{*} p<.017 ;{ }^{* *} p<.01$. 
Tabla 6. Continuación.

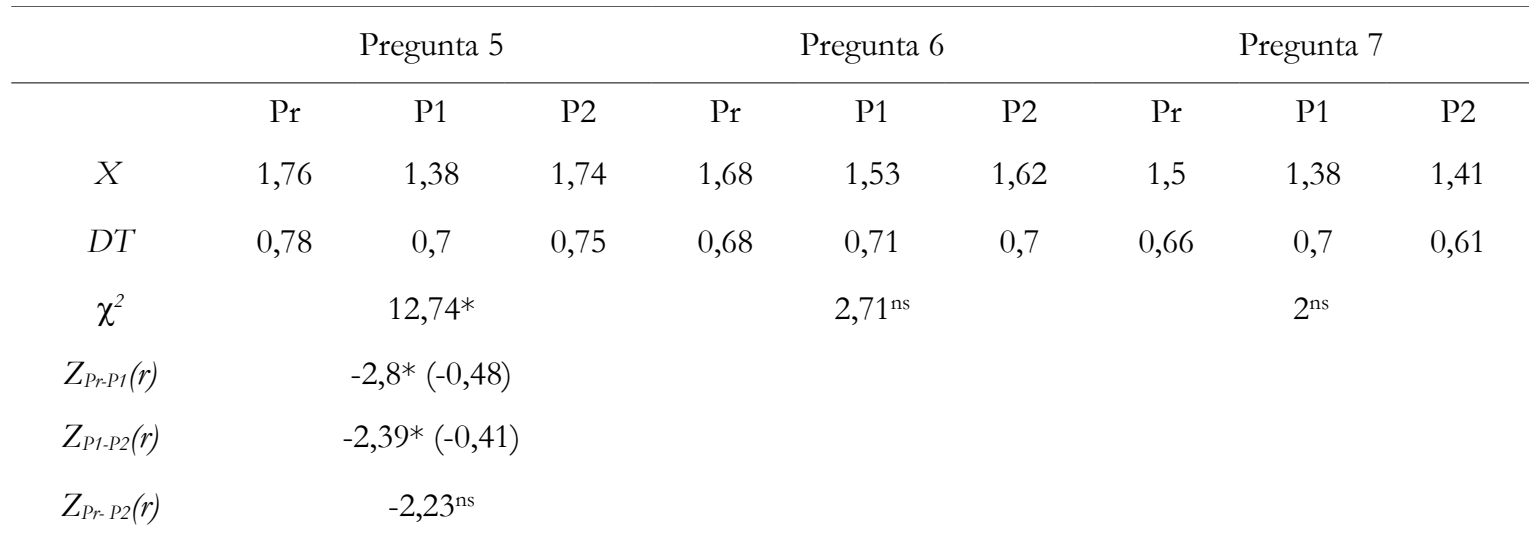

Pr: evaluación previa; P1: postest 1 (inmediato tras la experiencia); P2: postest 2 (dos meses después de la expe riencia); $X$ : media; DT: desviación típica; $\chi^{2}$ : estadístico ji-cuadrado; $Z$ : aproximación a lo normal; $r$ : tamaño del efecto, ns: no significativo; ${ }^{*} p<.017 ;{ }^{*} p<.01$.

\section{Contraste entre grupos}

Sobre los hábitos de higiene, en la primera toma de datos no se perciben diferencias estadísticamente significativas entre el grupo control y experimental; ambos grupos parten de los mismos hábitos y por tanto son grupos comparables. Tampoco hay diferencias estadísticamente significativas tras la intervención (Tabla 7). Sin embargo, sí se observan valores más altos en la tercera toma de datos para las preguntas 1-3 a favor del grupo control. Los datos obtenidos de las declaraciones de los niños indican que el método tradicional ha sido más efectivo a largo plazo respecto a los hábitos higiénicos.

Este resultado es contrario a lo obtenido por Faccio et al. (2013) y Mafra et al. (2015) con alumnado de Educación Primaria. Tales estudios consiguen mejores resultados en la introducción de hábitos relativos a la higiene alimentaria el primero y bucal el segundo, cuando el alumnado lleva a cabo procesos activos frente a los de tipo teórico o transmisivo. No obstante, ambos trabajos recogen exclusivamente información del alumnado inmediatamente después de la intervención, no estudian la permanencia en el tiempo de su efecto. En la intervención didáctica que se analiza, tanto el grupo experimental como el control realizaron las mismas actividades de aprendizaje sobre el lavado de manos y dientes (Anexo 1), la diferencia se encuentra en que el grupo experimental realizó además un proceso de investigación, mientras que el grupo control realizó dos fichas. Las fichas se almacenan y pueden contribuir al recuerdo de la información recibida. Esta hipótesis se ve apoyada por la similitud entre los últimos dibujos del grupo control (manos que se lavan para evitar los microbios) y una de las fichas realizadas.

Tabla 7. Contraste de las respuestas entre el grupo control y experimental

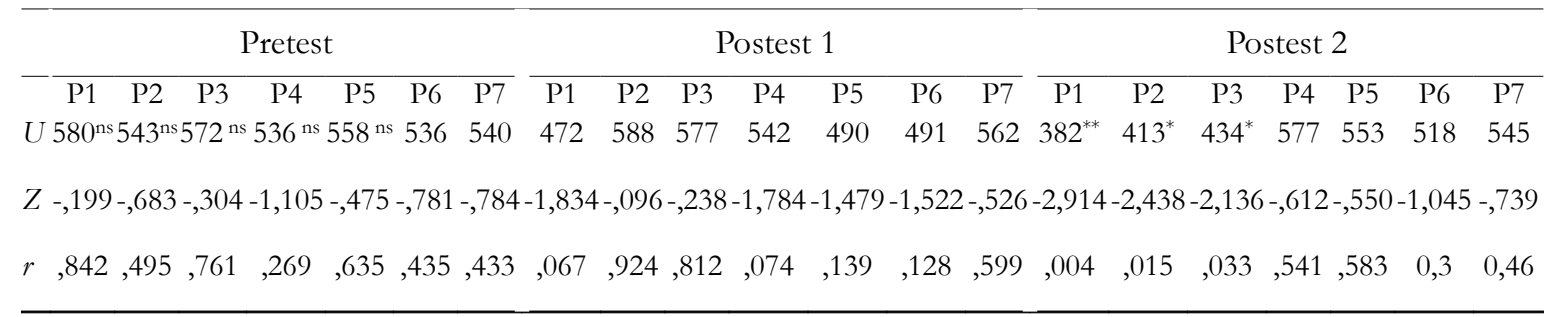

$U$ : estadístico de Mann-Whiney, $W$ : estadístico de Wilcoxon, $Z$ : aproximación a lo normal

La Tabla 8 muestra el contraste entre ambos grupos para el vocabulario en cada una de las aplicaciones. Solo aparecen diferencias estadísticamente significativas referentes a la palabra 
hongos. En el grupo control es mayor el número de individuos que afirman conocer el término tras la intervención didáctica; sin embargo, esta diferencia desaparece con el tiempo.

Veraksa et al. (2016), basándose en Vygotsky, afirman que, para el desarrollo de conceptos científicos, el encuentro comunicativo entre los maestros y los niños es fundamental. El mecanismo de aprendizaje primordial es, según este enfoque, la comunicación, más que, por ejemplo, la exploración. Esto podría explicar que no se hayan encontrado diferencias significativas entre ambas metodologías respecto a la adquisición de vocabulario o conceptos, puesto que en ambos casos los procesos comunicativos entre maestras y alumnos son muy similares. En el grupo experimental la profesora guía y orienta durante la experimentación, de acuerdo con Yoon y Onchwari (2006).

Sin embargo, es llamativo el caso de una niña del grupo experimental que desconocía el término hongo inicialmente pero que en la segunda aplicación declara: «Los hongos son microbios» y en la tercera: «Los hongos son cositas marrones que salen en las placas». De igual modo esta misma alumna, que desconoce inicialmente la palabra bacteria, dice tras la intervención: «Las bacterias son microbios» y dos meses después: «Son unas cosas pequeñitas que lo pones en las placas, los dedos, y salen».

Tabla 8. Contraste de las respuestas sobre las palabras conocidas entre el grupo control y experimental.

\begin{tabular}{|c|c|c|c|c|c|c|c|c|c|c|c|c|c|c|c|}
\hline & \multicolumn{3}{|c|}{ Microorganismos } & \multicolumn{3}{|c|}{ Microbios } & \multicolumn{3}{|c|}{ Virus } & \multicolumn{3}{|c|}{ Hongos } & \multicolumn{3}{|c|}{ Bacterias } \\
\hline & $\operatorname{Pr}$ & P1 & P2 & $\operatorname{Pr}$ & P1 & P2 & $\operatorname{Pr}$ & P1 & P2 & $\operatorname{Pr}$ & P1 & P2 & $\operatorname{Pr}$ & P1 & P2 \\
\hline$\chi^{2}$ & ns & ns & ns & $0,75^{\mathrm{ns}}$ & ns & ns & $1,9^{\text {ns }}$ & ns & $1,27^{\mathrm{ns}}$ & ns & $6,7^{* *}$ & ns & ns & $0,38^{\mathrm{ns}}$ & $0,05^{\text {ns }}$ \\
\hline$V$ & & & & & & & & & & & 0,46 & & & & \\
\hline
\end{tabular}

Pr: evaluación previa; P1: postest 1 (inmediato tras la experiencia); P2: postest 2 (dos meses después de la experiencia); $\chi 2$ : estadístico ji-cuadrado (cuando no aparece implica que se ha utilizado el estadístico de Fisher, por haber vulnerado el mínimo del $25 \%$ de casillas con recuento inferior a 5); $V$ : tamaño del efecto, mediante el estadístico de Cramer; ns: no significativo; ${ }^{* *} p<0.01$

Tal y como señalan Vivaldi y Salsa (2017), en los dibujos de los niños se pudo ver la evolución de los conceptos mediada por el proceso comunicativo con el docente y los pares. Destaca especialmente la adopción del bacteriófago como referente de microorganismo, su representación en plastilina ha tenido una influencia mucho mayor que la visualización de variedad de formas microbianas en una imagen proyectada. A pesar de no ser el tipo de virus que afecta a los humanos, fue el elegido por la maestra para modelar. La influencia de esta actividad apoya tanto la defensa tradicional de las actividades manipulativas en EI como la globalización de la enseñanza en la etapa, o según la denominación actual el enfoque STEAM integrado; porque una correcta integración de la enseñanza artística contribuye a mejorar el aprendizaje científico (Bequette y Bequette 2012).

\section{Análisis del cuestionario a padres}

Se recogieron 39 de los 69 cuestionarios facilitados a las familias (22 en el grupo experimental y 17 en el grupo control). Se realizó una tabla de contingencia entre las preguntas independientes: cambio en el hábito de lavarse los dientes y cambio en el lavado de manos. Según la prueba de Fisher ( $\mathrm{p}>.05$ en ambos casos), no hay diferencias entre el grupo experimental y el grupo control.

En el grupo experimental, un 18,18\% de los niños mencionaron a sus padres la importancia del lavado de dientes y un 13,64\% lo hicieron sobre la relevancia del lavado de manos. Solo un niño hizo referencia a que los microorganismos son seres vivos malos. En el grupo control, la 
mayoría de los padres expresó que sus hijos les habían hablado de la importancia de lavarse las manos $(29,41 \%)$ y sobre unos «seres vivos malos» $(17,65 \%)$.

Asimismo, las familias aportaron datos sobre lo que los niños espontáneamente habían contado acerca de la experiencia. Casi la mitad de los niños del grupo experimental (45,45\%) hablaron en casa de la actividad realizada con las placas Petri, sin embargo, no se registran respuestas sobre la actividad de elaboración de masa de pan.

Tanto los dibujos post-intervención del grupo experimental como la información aportada por las familias muestran el impacto de la pequeña investigación llevada a cabo con placas Petri. Este hallazgo rebate la recomendación de distintos autores de emplear actividades y materiales cotidianos en la enseñanza de las ciencias por su efectividad. Los resultados parecen indicar que algo desconocido para los niños, como los cultivos microbianos, ha generado mayor motivación y recuerdo que la realización de masa de pan con y sin levadura. Una de las posibles explicaciones es que en las placas de Petri hicieron visible lo invisible; la diversidad de formas, tamaños y colores de las colonias llamaron mucho la atención de los niños potenciando su curiosidad. Por otro lado, el sostenimiento de la actividad en el tiempo -dos semanas de observación y registro sobre una tabla- es sin duda otro factor importante.

Así mismo, estos resultados no concuerdan con la falta de diferencias estadísticas al comparar la permanencia en el tiempo de ambas metodologías (Tablas 8 y 9). Consideramos que se debe a que la evaluación previa y posterior a la intervención se ha limitado a conocimientos de tipo declarativo. No ha sido evaluada la curiosidad y su transformación en interés por aprender, ni otras capacidades científicas procedimentales o axiológicas. Surge así la necesidad de hacer una llamada de atención sobre el empleo de estrategias de razonamiento profundo y de comunicación de ideas entre los iguales durante y tras la realización de actividades de indagación, de modo que se evalúen más dimensiones del conocimiento científico, así como las emociones en juego.

\section{Conclusiones, limitaciones y prospectiva del estudio}

Los resultados de esta investigación resultan relevantes como contribución a la generación del corpus teórico en la Didáctica de las ciencias en la etapa de EI cuyo desarrollo, tanto en España como en el resto del mundo, es aún incipiente. El trabajo presentado no solo supone una aportación al conocimiento didáctico basado en pruebas, sino que muestra el interés de hacer un seguimiento de los aprendizajes en el tiempo.

Así mismo, la temática tratada y los resultados obtenidos tienen implicaciones educativas claras. Por un lado, animan a incluir sistemáticamente el estudio de los microorganismos en esta etapa ligado tanto a la adquisición de hábitos de higiene como al desarrollo de conceptos científicos tales como ser vivo o ecosistema y a la comprensión de las interrelaciones entre la ciencia, la tecnología y la sociedad.

Sin duda este trabajo también abre nuevos interrogantes que es necesario explorar. Las expresiones referidas a los microorganismos empleadas por los niños nos llevan a plantear nuevas prospectivas de investigación apoyadas en la teoría sociocultural de Vygotsky (2000); según la cual, la comunicación con los demás antecede al pensamiento. De este modo, surge la hipótesis de que los mensajes transmitidos por las familias y los equipos educativos de la primera etapa de EI (0-3 años) estarían forjando los hábitos de higiene y, al mismo tiempo, unos primeros modelos mentales con relación a los microorganismos y a la transmisión de enfermedades infecciosas, mucho antes de que comience la enseñanza reglada con relación a los mismos. De igual modo, se hace necesario conocer qué vocabulario utilizan los adultos cuando hablan con los niños y se refieren a los microrganismos. Los resultados de este trabajo 
muestran que algunos niños vinculan a los virus con la causa de enfermedades. Pero, ante la sospecha de que «bichos» es el vocablo más frecuente al referirse a los microorganismos, se recomienda el empleo de terminología científica precisa en las aulas y en los hogares, al igual que ya es habitual en la educación sexual. De este modo, se contribuirá a sentar las bases de una adecuada educación para la salud y a un mejor conocimiento científico.

En cuanto al contraste entre una metodología más tradicional y transmisiva y otra experimental con indagación, los datos extraídos tras la intervención no son concluyentes. El análisis de estos muestra la importancia de no limitarse al lenguaje oral en esta etapa educativa y el interés de la expresión plástica, tanto en volumen como sobre el papel. La intervención didáctica de tipo tradicional mostró a medio plazo una influencia positiva sobre el hábito de lavado de manos antes de comer que no se observó en el grupo experimental. Esta diferencia pudo estar mediada por el empleo de fichas de trabajo que no se realizaron en el grupo experimental. El proceso de indagación no parece haber tenido proyección en la adquisición de hábitos de higiene a pesar de ofrecer pruebas que los sustentan; tampoco en la ampliación o adquisición de conocimientos de microbiología. Con todo, es interesante subrayar el hecho de que dos meses después de la intervención, casi la mitad del alumnado del grupo experimental recordaba la actividad de indagación llevada a cabo durante dos semanas con materiales no cotidianos. Este hecho parece ligar la memoria a la emoción y podría estar contribuyendo a aprendizajes sobre la ciencia en sí, la naturaleza de la ciencia y sus prácticas epistémicas, aspecto sobre el que habría que seguir investigando.

\section{Disponibilidad de los datos}

Los autores ponen a disposición los datos que respaldan las conclusiones de este estudio, previa solicitud razonable.

\section{Agradecimientos}

Los autores quieren agradecer la colaboración del CEIP Alcalde Galindo (Chinchilla de Monte Aragón) donde se realizó el estudio, especialmente a las profesoras de EI, el alumnado y sus familias.

\section{Referencias}

Armitage E., Allen M.L. (2015) Children's Picture Interpretation: Appearance or Intention? Developmental Psychology, 51(9), 1201-1215. DOI: 10.1037/a0039571

Ballesteros M. I., Paños E., Ruiz-Gallardo J. R. (2018) Los microorganismos en la educación primaria: ideas de los alumnos de 8 a 11 años e influencia de los libros de texto. Enseñanza de las ciencias, 36(1), 79-98. https://ensciencias.uab.es/article/view/v36-n1ballesteros-panos-ruiz/2274-pdf-es

Bandiera M. (2007) Microorganisms: Every day knowledge predates and contrasts with school knowledge. En Pintó, R. y Couso, D. (Eds.), Contributions from science education research, (pp. 213-224). Dordrecht (The Netherlands): Springer

Bequette J.W, Bequette M.B. (2012) A Place for ART and DESIGN Education in the STEM Conversation. Art Education, 65(2), 40-47.

Byrne J. (2011) Models of Micro-Organisms: Children's knowledge and understanding of micro-organisms from 7 to 14 years old. International Journal of Science Education, 33(14), 1927-1961.

Byrne J., Grace M., Hanley P. (2009) Children's anthropomorphic and anthropocentric ideas about microorganisms: Educational research. Journal of Biological Education, 44(1), 37-43. 
Byrne J., Sharp, J. (2006) Children's ideas about micro-organisms. School Science Review, 88(322), 71-79. https://dialnet.unirioja.es/servlet/articulo?codigo $=2104605$

Carey S. (1985) Chonceptual change in childhood. Cambridge MIT Press.

Carver S. M., Shrager J. (Eds) (2012) The journey from child to scientist. Integrating cognitive development and the Education Sciences. Washington, D.C.: American Psychological Association.

Confederación de Sociedades Científicas de España COSCE (2011) Informe Enciende. Enseñanz̧a de las Ciencias en la Didáctica Escolar para edades tempranas en España. Madrid: COSCE

Cruz-Guzmán M., García-Carmona A., Criado A. M., (2017) Aprendiendo sobre los cambios de estado en educación infantil mediante secuencias de pregunta-prediccióncomprobación experimental. Enseñanza de las Ciencias, 35(3), 175-193. https://ensciencias.uab.es/article/view/v35-n3-cruz-guzman-garcia-criado/2336-pdfes

Díaz R., López R., García A., Abuín G., Nogueira E., García J.A. (1996) ¿Son los alumnos capaces de atribuir a los microorganismos algunas transformaciones de los alimentos? Enseñanza de las ciencias, 14(2), 143-153.

Dominguez C. R. C., Leporo N., De Franco M. T., Inglez G. C., Gonçalvez V.M., Bizerra A.F. (2018) Learning about Microorganisms in Childhood: Four to Six Year Old Children's Voice in Kindergartens and Museums. Revista Brasileira de Pesquisa em Educação em Ciências, 1(2), 1-25. https://periodicos.ufmg.br/index.php/rbpec/article/view/4583/2988

Eshach H. (2011) Science for young children: A new frontier for science education. Journal of Science Education \& Technology, 20, 435-443.

Eshach H., Fried M.N. (2005) Should science be taught in early childhood? Journal of Science Education \& Technology, 14, 315-336.

Faccio E., Costa N., Losasso C., Cappa V., Mantovani C., Cibin V., ... Ricci A. (2013) What programs work to promote health for children? Exploring beliefs on microorganisms and on food safety control behavior in primary schools. Food Control, 33(2), 320-329.

French L. (2004) Science as the center of a coherent, integrated early childhood curriculum. Early Childhood Research Quarterly, 19, 138-149.

Froschauer L. (2016) Early childhood physical science. Science and Children, 53(5), 5.

Gallegos-Cázares L., Flores-Camacho F., Calderón-Canales E., Posada de la Concha J.M. (2017) Representations over the Earth's shape and the process of day and night from Nahua indigenous schoolchildren / Representaciones sobre la forma de la Tierra y del proceso día y noche en niños de escuelas indígenas Nahuas, Infancia y Aprendizaje, 40(2), 343-380, DOI: 10.1080/02103702.2017.1292683

Garrido M. (2007) La evolución de las ideas de los niños sobre los seres vivos [Tesis doctoral]. Universidad de A Coruña, A Coruña.

Gerde H. K., Schachter R. E., Wasik B. A. (2013) Using the scientific method to guide learning: An integrated approach to early childhood curriculum. Early Childhood Education Journal, 41(5), 315-323.

Gómez-Motilla C., Ruiz-Gallardo J. R. (2016) El rincón de la ciencia y la actitud hacia las ciencias en educación infantil. Revista Eureka sobre enseñanza y divulgación de las ciencias, 13(3), 643-666. https://revistas.uca.es/index.php/eureka/article/view/2996 
Jones M. G., Rua M. J. (2006) Conceptions of germs: Expert to novice understandings of microorganisms. Electronic Journal of Science Education, 10(3). http://wolfweb.unr.edu/homepage/crowther/ej se/ejsev10n3.html

Kalish C.W. (1996) Preeschoolers' understanding of germs as invisible mechanisms. Cognitive Devolpment, 11, 83-106.

Kvale S. (2011) Las entrevistas en investigación cualitativa. Madrid: Morata.

Luna, M. y Solís, E. (1997). Las ideas previas del alumnado en Ciencias: una recopilación sobre los núcleos de contenidos del primer ciclo de la ESO. Título abierto: revista del CEP de Sevilla, 3, 63-74.

Mafra P., Lima N., Carvalho G, S. (2015) Experimental Activities in Primary School to learn about microbes in an Oral Health Education Context. Journal of Biological Education, 49(2), 190-203.

Mayerhofer N. (2009) La influencia de la palabra microbio en las representaciones iniciales de alumnos de primaria. Enseñanza de las ciencias: revista de investigación y experiencias didácticas, (Extra), 2073-2079.

Moreno F. J. (2015) Diseño y validación de un cuestionario para determinar los hábitos y conocimientos en bigiene corporal infantil, y su aplicación en escolares y menores en desamparo de la Región de Murcia. Tesis doctoral. Universidad de Murcia. http://hdl.handle.net/10201/47830

Piaget J. (2008) La representación del mundo en el niño. (10ª ed.). Madrid: Morata. (1973)

Prokop P., Fančovičová J., Krajčovičová A. (2016) Alternative Conceptions about Microorganisms are Influenced by Experiences with Disease in Children, Journal of Biological Education, 50(1), 61-72, DOI: 10.1080/00219266.2014.1002521

Portela C. (2013) De ingrediente de la comida a 'cosa asquerosa': percepción de los alumnos de Educación Básica de dos escuelas de São Gonçalo (Rio de Janeiro, Brasil) sobre los insectos. Boletín de la Sociedad Entomológica Aragonesa (S.E.A.), 52, 317-321.

Real Decreto 114/2004, de 23 de enero, por el que se establece el currículo de la Educación Infantil. Boletin Oficial del Estado. Madrid, 6 de febrero, 32, 5041-5050.

Ruiz-Gallardo J. R., Paños E. (2018) Primary school students' conceptions about microorganisms. Influence of theoretical and practical methodologies on learning. Research in Science \& Technological Education, 36(2), 165-184.

Saçkes M., Trundle K. C., Bell R. L., O'Connell A. A. (2011) The influence of early science experience in kindergarten on children's immediate and later science achievement: Evidence from the early childhood longitudinal study. Journal of Research in Science Teaching, 48(2), 217-235.

Sigelman C. K., Glaser S. E. (2019) Characterizing children's intuitive theories of disease: The case of flu. Cognitive Development 100809. https://doi.org/10.1016/j.cogdev.2019.100809

Veraksa N., Shiyan O., Shiyan I., Pramling N., Pramling-Samuelsson I. (2016) Communication between teacher and child in early child education: Vygotskian theory and educational practice / La comunicación entre profesor y alumno en la educación infantil: la teoría vygotskiana y la práctica educativa, Infancia y Aprendizaje, 39(2), 221-243. doi: 10.1080/02103702.2015.1133091 
Vijapurkar, J., Konde P. (2014) «Omne Vivum Ex Vivo»? A Study of Middle School Students' Explanations of the Seemingly Sudden Appearance of Some Life Forms. Research in Science Education, 44, 885-902. https://doi.org/10.1007/s11165-014-9406-1

Vivaldi, Salsa (2017) Drawing for others: influence of referential intention in early production of drawings / Dibujar para otros: influencia de la intencionalidad referencial en la producción temprana de dibujos, Infancia y Aprendizaje, 40(1), 56-87, DOI: 10.1080/02103702.2016.1263453

Vygotsky L. S. (2000) Pensamiento y lenguaje. Barcelona: Paidós

Watters J.J., Diezmann C.M., Grieshaber S.J., Davis J.M. (2000) Enhancing science education for young children: A contemporary initiative. Australian Journal of Early Childhood, 26(2), 1-7.

Westcott H.L. Littleton K.S. (2005) Exploring meaning in interviews with children. En S. Greene \& D. Hogan (Eds), Researching children's experience (pp. 141-157). London: Sage

Yoon J. Onchwari J. A. (2006) Teaching young children science: Three key points. Early Childhood Education Journal, 33(6), 419-423. 
Anexo 1. Resumen de la secuencia didáctica seguida con los dos grupos

\section{GRUPO EXPERIMENTAL}

AMBOS GRUPOS

GRUPO CONTROL

1. Dibujo de un microorganismo en un entorno adecuado + cuestionario inicial ( $1^{2}$ TOMA DE DATOS $)$ 2. Elaboración de masa de pan por grupos con y sin levadura

3. Observación de resultados y conclusiones en asamblea
2. Visualización de vídeo sobre cómo se elabora la masa del pan

3. Ficha 1: Rodear qué ingrediente hace crecer la masa de pan

4. Explicación con apoyo TIC: aspecto real de los microbios, tipología y relación con el ser humano (beneficiosa y perjudicial), importancia de las vacunas y la higiene

5. Realización de un bacteriófago con plastilina

6. Tutorización del lavado correcto de manos y dientes Juego simbólico: lavado de dientes con maqueta de la boca

7. ¿Es importante la higiene? Canción 'A lavarse las manos'

(Indagación)

Presentación de las placas de Petri con medio de cultivo

Experimentación 4 grupos habituales

de clase: Lavado de manos (sí/no);

Lavado de dientes (sí/no)

Observación y registro durante 2

semanas

Discusión de los resultados finales
7. Ficha 2: Ordenan la secuencia lógica del lavado de dientes y dibujan los elementos necesarios para lavar las manos

8. Dibujo de un microorganismo en un entorno adecuado + cuestionario (2 $2^{\mathrm{a}}$ TOMA DE DATOS) 
Anexo 2. Cuestionario higiene oral e higiene de manos

$$
\text { Nombre: }
$$

Curso: Edad:

¿Te lavas las manos antes de cada comida?

$\square$ Siempre $\square$ A veces $\square$ Nunca

¿Te lavas las manos después de hacer caca?

$\square$ Siempre $\square$ A veces $\square$ Nunca

¿Te lavas las manos después de hacer pipí?

$$
\text { Siempre } \square \text { A veces } \square \text { Nunca }
$$

¿Usas jabón cuando te lavas las manos?

$\square$ Siempre $\square$ A veces $\square$ Nunca

$¿$ Te lavas los dientes al levantarte?

$\square$ Siempre $\square$ A veces $\square$ Nunca

¿Te lavas los dientes después de comer?

$\square$ Siempre $\square$ A veces $\square$ Nunca

$¿$ Te lavas los dientes antes de ir a la cama?

$\square$ Siempre $\square$ A veces $\square$ Nunca

¿Has oído alguna vez la palabra microorganismo? y ¿microbio?, ¿virus?, ¿hongos?, ¿bacterias? ¿sabes lo que es? 
Anexo 3. Cuestionario para las familias

Con el fin de potenciar hábitos saludables en los niños/as, concretamente aquellos relacionados con la higiene bucal y de las manos, hemos realizado una serie de actividades y quisiéramos saber su repercusión en su comportamiento habitual. Para ello, le agradeceríamos que contestara a este breve cuestionario y se lo facilite a su hijo para que nos lo devuelva.

Reciba un cordial saludo.

¿Le ha hablado su hijo/a de la actividad de microbios que se ha realizado? (En el caso del grupo control, la pregunta es: ¿Le ha comentado su hijo/a algo sobre el tema de los microbios?)

Sí

¿Qué cosas le ha contado?

¿Su hijo/a se lava los dientes?

\begin{tabular}{|c|c|}
\hline 呈 & Nunca \\
\hline 豆 & Cuando lo obligamos \\
\hline 哣 & Voluntariamente \\
\hline & Solo a veces \\
\hline
\end{tabular}

¿Ha notado algún cambio en el hábito de lavado de dientes de su hijo/a?

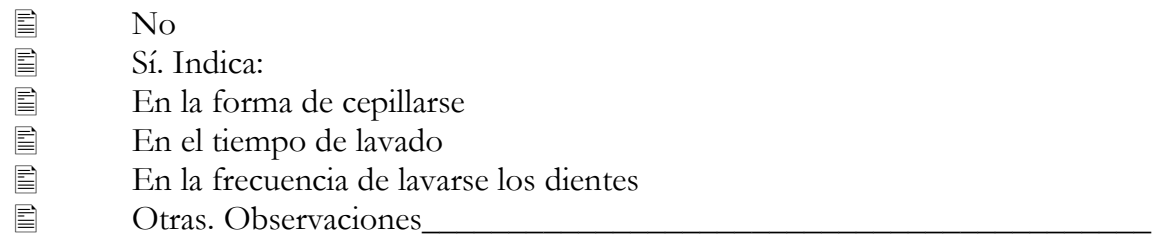

¿Su hijo/a se lava las manos?

\begin{tabular}{|c|c|}
\hline 奜 & Nunca \\
\hline & Cuando lo obligamos \\
\hline & Voluntariamente \\
\hline & Solo a veces \\
\hline
\end{tabular}

¿Ha notado algún cambio en el hábito de lavado de manos de su hijo/a?

\begin{tabular}{|c|c|}
\hline 帠 & No \\
\hline 豆 & Sí. Indica: \\
\hline 产 & En la forma de lavarse \\
\hline 豆 & En el tiempo de lavado \\
\hline 豆 & En la frecuencia de lavarse las manos \\
\hline 辰 & Otras. Observaciones \\
\hline
\end{tabular}

